\title{
Photothermal method with arbitrary phase shift
}

by A. FIGARI (

(*) CISE Tecnologie Innovative, Via Reggio Emilia 39, 20090 Segrate (Milano), Italy.

\begin{abstract}
An intensity-modulated pump beam generates a thermal wave in a sample. The relationship between the phase of the photothermal signal and the thermal wavelength $\lambda_{\text {th }}$ is pointed out, as the probe beam explores the thermal wave. On the basis of this analysis, the wavelength and the thermal diffusivity $\alpha$ of a sample can be measured with a new experimental procedure which uses arbitrary signal phase shifts relative to the heating spot centre. An application is reported.
\end{abstract}

\section{Nomenclature}

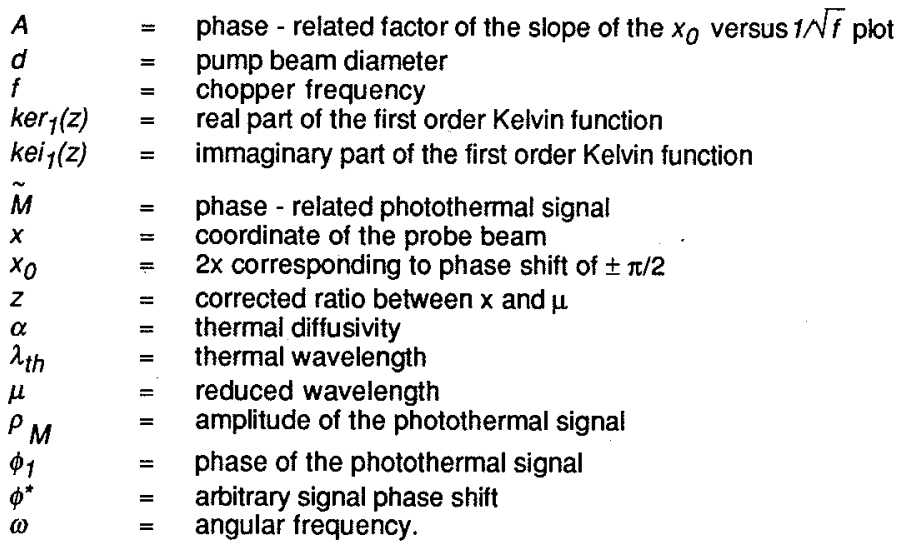

\section{Introduction}

The photothermal deflection technique $[1,2]$ provides a valuable spectroscopic and thermal diagnostic tool. In particular, absolute and noncontact measurements of the thermal diffusivity $\alpha$ of materials can be made [3,4].

A radiation beam (pump beam) is intensity-modulated with frequency $\omega=2 \pi f$ using a chopper, and then focussed to a tiny region of the sample surface (figure 1 ). A thermal wave is then generated in the sample, as described by the solution of the periodical diffusion equation, exhibiting the behaviour of a strongly damped wave (see, e.g., reference [5]). The thermal wavelength is defined as:

$$
\lambda_{t h}=2 \pi\left(\frac{2 \alpha}{\omega}\right)^{1 / 2}
$$

The probe beam grazes along the sample surface: the distance $x$ from the beam to the centre of the heated spot can be varied. 


\section{http://dx.doi.org/10.21611/qirt.1992.059}

Once the position $x$ is fixed, the probe beam is periodically deflected, due to the thermal gradient in the air close to the sample, with the components of the deflection vector in directions both perpendicular and parallel to the surface. It is this latter component which is measured by a position sensor and a two-phase lock-in amplifier.

In the classic experiment $[3,4] \alpha$ is determined by measuring the separation $x_{0}$ between the points corresponding to a phase shift of $\pm \pi / 2$ with respect to the spot centre $x=0$.

Taking into account the pump beam diameter via the constant $d$, one can write:

$$
x_{0}=\frac{\lambda_{t h}}{2}+d
$$

and:

$$
x_{0}=\sqrt{\pi \alpha} f^{-1 / 2}+d
$$

According to equation (3), $\alpha$ can be obtained from the slope of the $x_{0}$ versus $1 / \sqrt{f}$ plot. In references [3] and [4] the experimental procedure was justified by heuristic considerations and supported by numerical experimentation, while an analytical foundation to this technique was given in reference [6].

Following this theory a new experimental scheme is suggested in this communication: $\lambda_{\text {th }}$ and $\alpha$ can be determined by measuring the separation $x_{0}\left(\phi^{*}\right)$ between the points corresponding to an arbitrary phase shift $\phi^{*}$ of the signal relative to the spot centre.

\section{Theory}

By defining the thermal diffusion length in the medium [7], or reduced wavelength [5]:

$$
\mu=\frac{\lambda_{t h}}{2 \pi}
$$

and putting:

$$
z=\frac{\sqrt{2} x}{\mu}
$$

the analysis developed in reference [6] shows that the photothermal signal can be described in terms of first order Kelvin functions; it is proportional to:

$$
\tilde{M}(z)=\tilde{\rho}_{M}(z) e^{i \phi_{0}}\left[k e r_{1}(z)-i k e i_{1}(z)\right]
$$

where $\tilde{\rho}_{M}(z)$ is an amplitude, $e^{i \phi_{0}}$ a constant phase factor.

$\tilde{M}(z)$ can be written as:

$$
\tilde{M}(z)=\rho_{M}(z) e^{i \phi_{0}} \exp \left[i \phi_{1}(z)\right]
$$

where $\rho_{M}(z)$ is an amplitude and the phase $\phi_{1}(z)$ a function which can be easily calculated: it is tabulated (e.g. in reference [8], page 433), and is shown in figure 2.

Relations (5) and (7) show that the phase $\phi_{1}(z)$ of the photothermal signal depends only 
on $z$, the corrected ratio of distance $x$ to $\mu$. If $\phi^{*}$ is the phase shift of the signal relative to the spot centre, from the definitions of $z$ [equation (5)], $\mu$ [equation (4)], $x_{0}(=2 x)$ and taking into account the pump-beam diameter via the constant $d$, one can write:

$$
x_{0}\left(\phi^{*}\right)=A\left(\phi^{*}\right) \frac{\lambda_{\text {th }}}{2}+\alpha
$$

with

$$
A\left(\phi^{*}\right)=\frac{\sqrt{2} z}{\pi}
$$

From the definition of $\lambda_{t h}$ [equation (1)], one obtains:

$$
x_{0}\left(\phi^{*}\right)=A\left(\phi^{*}\right) \sqrt{\pi \alpha} f-1 / 2+d
$$

The algorithm for obtaining the factor $A\left(\phi^{*}\right)$ is the following: $\phi^{*}(z)$ is arbitrarily fixed, equation (7) gives the corresponding value $z$ (reference [8] and figure 2 ) and equation (9) gives $A$.

\section{Results}

As an example, an application of the experimental technique to two metals is reported on. Figures 3 and 4 show the $x_{0}$ offset plotted versus $f^{-1 / 2}$ for different values of phase shift.

Table I reports the chosen phase shifts and the corresponding $A\left(\phi^{*}\right)$.

The results are plotted in figure 3 a) for gold (999.0) and 3 b) for copper (DHP 99.9\%).

The experimental values compare well with literature data, considering that the technique is a non-contact one: $\alpha=1.27 \mathrm{~cm}^{2} / \mathrm{s}$ for gold (literature value [9] $\alpha=1.30 \mathrm{~cm}^{2} / \mathrm{s}$ ), $\alpha=1.13 \mathrm{~cm}^{2} / \mathrm{s}$ for copper (literature value [9] $\alpha=1.16 \mathrm{~cm}^{2} / \mathrm{s}$ ).

However, a rigorous statistical analysis of the accuracy and resolution improvement compared with the method with fixed phase shift requires more advanced laboratory instruments than those used in these experiments, taking into account that computerized thermal wave microscopy should be the final goal of these techniques.

\section{Conclusion}

The analytical relationship describing the phase of the photothermal signal within the thermal wavelength is pointed out: it relates the phase to the thermal wavelength and diffusivity, permitting one to measure $\lambda_{t h}$ and $\alpha$ choosing arbitrary signal phase shifts relative to the spot centre. A reported application to metals is in good agreement with the theory.

\section{REFERENCES}

[1] BOCCARA (A.C.), FOURNIER (D.) and BADOZ (J.). - Thermo-optical spectroscopy: Detection by the "mirage effect". Appl. Phys. Lett., 36 (2), 1980, p. 130-132.

[2] MURPHY (J.C.) and AAMODT (L.C.). - Photothermal spectroscopy using optical beam probing: Mirage effect. J. Appl. Phys., 51 (9), 1980, p. 4580-4588.

[3] KUO (P.K.), LIN (M.J.), REYES (C.B.), FAVRO (L.D.), THOMAS (R.L.), KIM (D.S.), ZHANG (S.Y.), INGLEHART (L.J.), FOURNIER (D.), BOCCARA (A.C.) and YACOUBI (N.). - Mirage- 
effect measurement of thermal diffusivity. Part l: experiment. Can. J. Phys., 64 (9), 1986, p. $1165-1167$.

[4] KUO (P.K.), SENDLER (E.D.), FAVRO (L.D.) and THOMAS (R.L.) - Mirage effect measurement of thermal diffusivity. Part II: theory. Can J. Phys., 64 (9), 1986, p. 1168-1171.

[5] BUSSE (G.) and ROSENCWAIG (A.). - Subsurface imaging with photoacoustics. Appl. Phys. Lett. 36 (10), 1980, p. 815-816.

[6] FIGARI (A.). - Analytical relations between the phase of the photothermal signal and the thermal wavelength. J. Appl. Phys., 71 (7), 1992, p. 3138-3142.

[7] ROSENCWAIG (A.). - Photoacoustics and photoacoustic spectroscopy, 1980, Wiley Interscience, New York.

[8] ABRAMOVITZ (M.) and STEGUN (1.). - Handbook of mathematical functions, 8th ed., 1972, Dover, New York.

[9] TOULOUKIAN (L.R.), POWELL (R.W.), HO (C.Y.) and NICOLAOU (M.C.). - Thermal diffusivity, 1973, IFI/Plenum, New York.

\begin{tabular}{|l|l|}
\hline \multicolumn{2}{|c|}{ TABLE I } \\
\hline$\phi^{*}$ & $A\left(\phi^{*}\right)$ \\
\hline & \\
$-\pi / 4$ & 0.673 \\
$-(3 / 8) \pi$ & 0.940 \\
$-\pi / 2$ & 1.2 \\
$-(5 / 8) \pi$ & 1.457 \\
$-(3 / 4) \pi$ & 1.713 \\
\hline
\end{tabular}

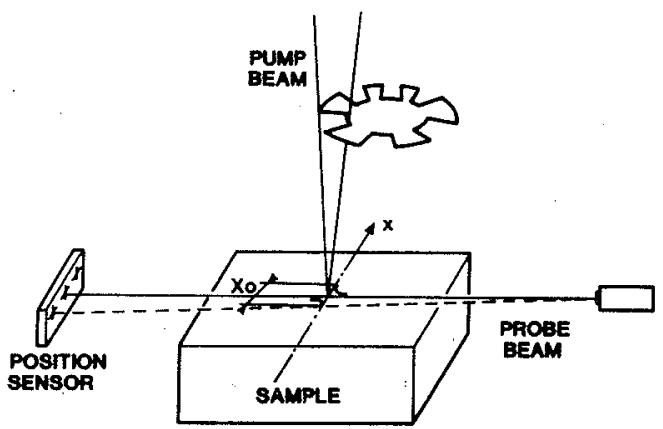

Fig. 1. - Experimental setup

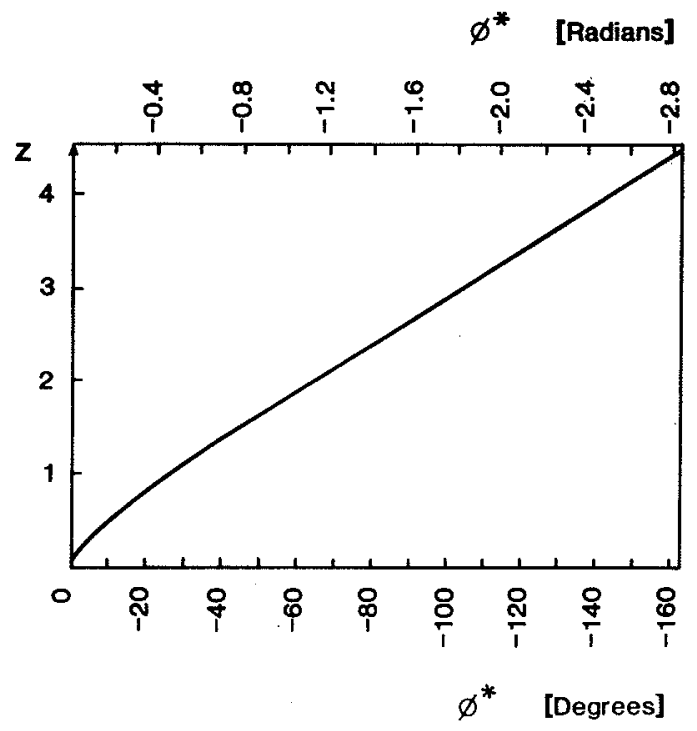

Fig. 2. - Relationship between the phase $\phi^{*}$ of the photothermal slgnal and $z$ 


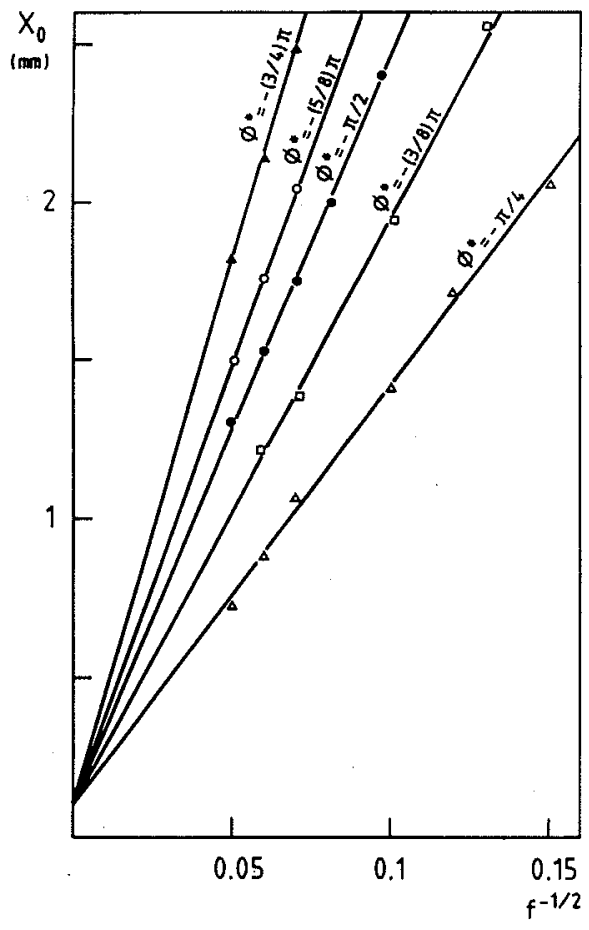

a)

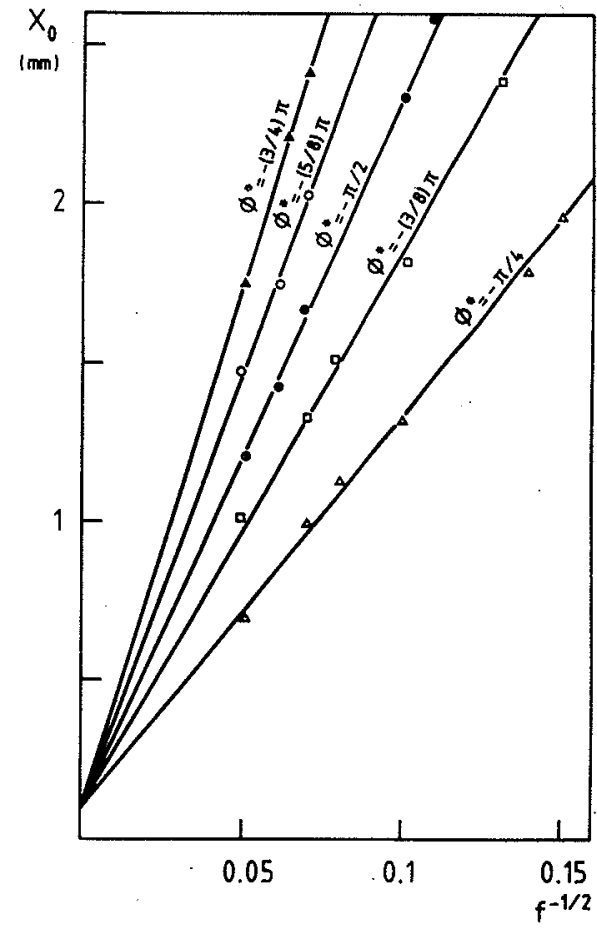

b)

Fig. 3. - Measurement of the thermal diffusivity: a) gold specimen (999.0); b) copper specimen (DHP 99.9\%). The value of $\alpha$ is obtalned from the angular coefficient of the stralght lines.

The experimental values corresponding to some phase shifts are reported in the following table :

$\begin{array}{llll} & & \text { gold } & \text { copper } \\ \begin{array}{l}\text { Straight } \\ \text { Iine }\end{array} & \phi^{*} & \alpha\left(\mathrm{cm}^{2} / \mathrm{s}\right) & \alpha\left(\mathrm{cm}^{2} / \mathrm{s}\right) \\ \text { empty triangle } & -\pi / 4 & 1.25 & 1.09 \\ \begin{array}{l}\text { square } \\ \text { full circle }\end{array} & -(3 / 8) \pi & 1.25 & 1.11 \\ \text { empty circle } & -\pi / 2 & 1.26 & 1.11 \\ \text { full triangle } & -(5 / 8) \pi & 1.29 & 1.14 \\ & -(3 / 4) \pi & 1.28 & 1.18\end{array}$

For gold the mean value is $\alpha=1.266 \cong 1.27 \mathrm{~cm}^{2} / \mathrm{s}$ (Ilterature value: $\alpha=1.30 \mathrm{~cm}^{2} / \mathrm{s}$ ).

For copper the mean value is $\alpha=1.126 \cong 1.13 \mathrm{~cm}^{2} / \mathrm{s}$ (IIterature value: $\alpha=1.16 \mathrm{~cm}^{2} / \mathrm{s}$ ). 